March 1995

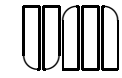

UMDEPP 95-113

\title{
A Theory of Spinning Particles for Large N-extended Supersymmetry
}

\author{
S. James Gates, Jr.1, 6 and L. Rana \\ Department of Physics \\ University of Maryland \\ College Park, MD 20742-4111 USA \\ gates@umdhep.umd.edu \\ lubna@umdhep.umd . edu
}

\begin{abstract}
An explicit construction of theories of spinning particles, both massive and massless, is given with arbitrary extended supersymmetry on the world-line. As an application of our results, we give a universal description of 3D (and via truncation all lower dimensional) supersymmetric scalar multiplets with arbitrary N-extended supersymmetry.
\end{abstract}

\footnotetext{
${ }^{1}$ Supported in part by National Science Foundation Grant PHY-91-19746

${ }^{2}$ Supported in part by NATO Grant CRG-93-0789
} 


\section{Introduction}

The simplest of supersymmetric systems are those that involve a single bosonic dimension. The best known example of this is the spinning particle [1]. The uses of such systems is surprisingly broad given their simplicity. Some of these include simulation of more complicated spinning strings [2], calculation of field theory anomalies [3], possible realization of new Kac-Moody algebras [4] and as generating functions for superintegrable systems like SKdV [5]. Despite all of these applications, a search of the literature reveals that almost no work has been done in elucidating the general structure of such theories. This will be the purpose of this work.

In the following we will give an explicit construction of the spinning particle action (both massive and massless) for all values of N-extended supersymmetry. We show that a basis for all such theories is provided by certain real representations of generalized Pauli-matrix algebras. As an application of our work, we construct a universal description of on-shell scalar multiplets with arbitrary N-extended supersymmetry in three or less bosonic dimensions.

\section{Minimal 1D, N-Extended Supergravity}

In this section, we introduce the concept of a minimal 1D representation of the supergravity multiplet that is valid for arbitrary (and large) values of $\mathrm{N}$, the degree of extended supersymmetry. The supersymmetry variations of the $1 \mathrm{D}, \mathrm{N}$-extended worldline supergravity fields may be defined on $\left(e, \chi^{\mathrm{I}}\right)$ where $e(\tau)$ is real and $\chi^{\mathrm{I}}(\tau)$ is a real spinor $($ with $\mathrm{I}=1, \ldots, \mathrm{N})$,

$$
\delta_{Q} e=-i 4 e^{2} \alpha^{\mathrm{I}} \chi^{\mathrm{I}} \quad, \quad \delta_{Q} \chi^{\mathrm{I}}=-\left(\partial_{\tau} \alpha^{\mathrm{I}}\right)
$$

The general coordinate transformations can be written as:

$$
\delta_{G . C} e=\left(\partial_{\tau} e\right) \xi-e\left(\partial_{\tau} \xi\right) \quad, \quad \delta_{G . C} \chi^{\mathrm{I}}=\partial_{\tau}\left(\chi^{\mathrm{I}} \xi\right)
$$

Now a direct calculation shows that

$$
\left[\delta_{Q}\left(\alpha_{1}\right), \delta_{Q}\left(\alpha_{2}\right)\right]=\delta_{G . C .}\left(\xi_{12}\right)+\delta_{Q}\left(\alpha_{12}{ }^{\mathrm{I}}\right)
$$

where $\xi_{12} \equiv i 4 e\left(\alpha_{1}{ }^{\mathrm{I}} \alpha_{2}{ }^{\mathrm{I}}\right)$ and $\alpha_{12}{ }^{\mathrm{I}} \equiv \xi_{12} \chi^{\mathrm{I}}$. Thus, it is not necessary to gauge a set of automorphisms (i.e. the generalization of the $\mathrm{U}(1)$ group seen in the $\mathrm{N}=2$ nonminimal theory) and (2.3) may be taken as the definition of the 1D local supersymmetry group for arbitrary numbers of real supercharges. 


\section{1D, N-Extended Rigid Supersymmetry and Matter}

Having begun with the representation of the supergravity multiplet for arbitrary values of $\mathrm{N}$, we next need to study the matter multiplets that are available to be coupled to these minimal worldline supergravity multiplets. In terms of real fields $\left(\phi_{i}, \psi_{\hat{j}}\right)$ with $i=1, \ldots, \mathrm{d}, \hat{i}=1, \ldots, \mathrm{d}$ we propose that the supersymmetry transformation laws for a scalar supermultiplet can be written as:

$$
\delta_{Q} \phi_{i}=i 2 \alpha^{K}\left(\mathrm{~L}_{K}\right)_{i}^{\hat{j}} \psi_{\hat{j}} \quad, \quad \delta_{Q} \psi_{\hat{i}}=\alpha^{K}\left(\mathrm{R}_{K}\right)_{\hat{i}}^{j} \partial_{\tau} \phi_{j}
$$

It is of importance that we have not made the a priori assumption that $\mathrm{d}=\mathrm{N}$. Note that the reality of the fields of necessity implies that the matrices $\left(\mathrm{L}_{K}\right)_{i}{ }^{\hat{j}}$ and $\left(\mathrm{R}_{K}\right)_{\hat{i}}{ }^{j}$ that appear above are also real. By requiring that the operator equation should read

$$
\left[\delta_{Q}\left(\alpha_{1}\right), \delta_{Q}\left(\alpha_{2}\right)\right]=i 4 \alpha_{1}^{K} \alpha_{2}^{K} \partial_{\tau}
$$

uniformly on both fields we find that the matrices $\mathrm{L}$ and $\mathrm{R}$ satisfy the following algebra:

$$
\mathrm{L}_{K} \mathrm{R}_{P}+\mathrm{L}_{P} \mathrm{R}_{K}=-2 \delta_{K P} \mathrm{I}, \mathrm{R}_{K} \mathrm{~L}_{P}+\mathrm{R}_{P} \mathrm{~L}_{K}=-2 \delta_{K P} \mathrm{I} .
$$

If the representations denoted by $i$ are distinguishable from those denoted by $\hat{i}$, it follows that a second distinct "twisted" scalar multiplet can be constructed. It is described by the set of supersymmetry variations given by

$$
\delta_{Q} \widehat{\phi}_{\hat{i}}=i 2 \alpha^{K}\left(\mathrm{R}_{K}\right)_{\hat{i}}^{j} \widehat{\psi}_{j} \quad, \quad \delta_{Q} \widehat{\psi}_{i}=\alpha^{K}\left(\mathrm{~L}_{K}\right)_{i}{ }^{\hat{j}} \partial_{\tau} \widehat{\phi}_{\hat{j}}
$$

Furthermore, it may happen that there are a number of inequivalent representations of the algebra in (3.3). For each such inequivalent representation, there would correspond a distinct scalar multiplet such as appears in (3.1) together with a twisted version such as in $(3.4)^{3}$.

For general values of $\mathrm{N}$, we can also construct spinor multiplets with the respective transformations laws,

$$
\delta_{Q} \eta_{\hat{i}}=\alpha^{K}\left(\mathrm{R}_{K}\right)_{\hat{i}}^{j} F_{j} \quad, \quad \delta_{Q} F_{i}=i 2 \alpha^{K}\left(\mathrm{~L}_{K}\right)_{i}{ }^{\hat{j}} \partial_{\tau} \eta_{\hat{j}}
$$

\footnotetext{
${ }^{3}$ This is the origin of the surprisingly large number of $(4,0)$ scalar and spinor multiplets found in [6].
} 
and twisted spinor multiplets

$$
\delta_{Q} \widehat{\eta}_{i}=\alpha^{K}\left(\mathrm{~L}_{K}\right)_{i}{ }^{\hat{j}} \widehat{F}_{\hat{j}} \quad, \quad \delta_{Q} \widehat{F}_{\hat{i}}=i 2 \alpha^{K}\left(\mathrm{R}_{K}\right)_{\hat{i}}^{j} \partial_{\tau} \widehat{\eta}_{j}
$$

Again the number of such distinct multiplets is in one-to-one correspondence with the number of inequivalent representation of the algebra in (3.3).

Before we move on to the question of invariant actions, it is extremely interesting to note that the existence of the $1 \mathrm{D}$, arbitrary N-extended supergravity multiplet was independent of the existence of the algebra in (3.3). The 1D supergravity formed a representation of the local $1 \mathrm{D}$ supersymmetry algebra in (2.3) without reference to the matrix algebra in (3.3). It is the existence of the matter field representations that depend on the latter algebra. We believe that this is an important lesson regarding representations of local supersymmetry in general. The local 1D supergravity multiplet need not be tied to any matrix realization of supersymmetry 4 ! On the other hand, the matter multiplets can be classified by precisely the number of inequivalent representations of the matrix representation.

For an invariant Lagrangian, we may take

$$
\mathcal{L}_{S M}=\left(\partial_{\tau} \phi_{i}\right)\left(\partial_{\tau} \phi_{i}\right)+i 2 \psi_{\hat{i}} \partial_{\tau} \psi_{\hat{i}}
$$

whose invariance requires the condition,

$$
\left(\mathrm{L}_{I}\right)^{i \hat{j}}+\left(\mathrm{R}_{I}\right)^{\hat{j} i}=0
$$

where the indices on the $\mathrm{L}$ and $\mathrm{R}$ matrices are raised with the use of Kronecker deltas.

In a similar manner, we can write an invariant Lagrangian for the spinor multiplet of the form,

$$
\mathcal{L}_{F M}=i 2 \eta_{\hat{i}} \partial_{\tau} \eta_{\hat{i}}+F_{i} F_{i}
$$

whose invariance leads to precisely the same condition as in (3.8). The same identity arises if one considers the twisted versions of the multiplets.

\section{1D, N-Extended Local Systems}

Having determined the general structure of 1D supergravity and as well the general structure of 1D supersymmetric scalar and spinor multiplets, it is now time to put

\footnotetext{
${ }^{4}$ It is tempting to believe that the present inability to find off-shell representations of $4 \mathrm{D}, N>4$ supergravity is somehow related to this fact.
} 
these two pieces together and investigate spinning particle theories. To begin the local version of (3.1) takes the form

$$
\delta_{Q} \phi_{i}=i 2 \alpha^{K}\left(\mathrm{~L}_{K}\right)_{i}^{\hat{j}} \psi_{\hat{j}} \quad, \quad \delta_{Q} \psi_{\hat{i}}=\alpha^{K}\left(\mathrm{R}_{K}\right)_{\hat{i}}^{j} \mathcal{D}_{\tau} \phi_{j}
$$

and leave invariant the action;

$$
\mathcal{S}_{S M}=\int d \tau\left[e^{-1} \mathcal{D}_{\tau} \phi_{i} \mathcal{D}_{\tau} \phi_{i}+i 2 e^{-1} \psi_{\hat{i}} \mathcal{D}_{\tau} \psi_{\hat{i}}+i 2 \chi^{K}\left(\mathrm{R}_{K}\right)_{\hat{i}}^{j} \psi_{\hat{i}} \mathcal{D}_{\tau} \phi_{j}\right]
$$

where

$$
\begin{aligned}
\mathcal{D}_{\tau} \phi_{i} & \equiv e\left[\partial_{\tau} \phi_{i}+i 2 \chi^{K}\left(\mathrm{~L}_{K}\right)_{i}{ }^{\hat{k}} \psi_{\hat{k}}\right], \\
\mathcal{D}_{\tau} \psi_{\hat{k}} & \equiv e\left[\partial_{\tau} \psi_{\hat{k}}+\chi^{K}\left(\mathrm{R}_{K}\right)_{\hat{k}}{ }^{i} \mathcal{D}_{\tau} \phi_{i}\right] .
\end{aligned}
$$

The local transformation laws for the spinor multiplet are given by

$$
\delta_{Q} \eta_{\hat{i}}=\alpha^{K}\left(\mathrm{R}_{K}\right)_{\hat{i}}^{j} F_{j} \quad, \quad \delta_{Q} F_{i}=i 2 \alpha^{K}\left(\mathrm{~L}_{K}\right)_{i}{ }^{\hat{j}} \mathcal{D}_{\tau} \eta_{\hat{j}}
$$

where

$$
\mathcal{D}_{\tau} \eta_{\hat{i}} \equiv e\left[\partial_{\tau} \eta_{\hat{i}}+\chi^{K}\left(\mathrm{R}_{K}\right)_{\hat{i}}^{k} F_{k}\right]
$$

An invariant action is given by

$$
\mathcal{S}_{F M}=\int d \tau\left[e^{-1} i 2 \eta_{\hat{i}} \mathcal{D}_{\tau} \eta_{\hat{i}}+e^{-1} F_{i} F_{i}+i 2 \chi^{K}\left(\mathrm{R}_{K}\right)_{\hat{i}}^{j} \eta_{\hat{i}} F_{j}\right]
$$

whose relevance will become clear below.

It is, of course, possible to consider these supermultiplets as representations of some additional symmetry groups. Under this circumstance each field of the multiplet carries an additional index $\left(\eta_{\hat{i}}, F_{i}\right) \rightarrow\left(\eta_{\hat{i}}^{\hat{\mathrm{I}}}, F_{i}^{\hat{\mathrm{I}}}\right)$. In the construction below, this property will be important. In particular, we can pick $\hat{\mathrm{I}}=i$, so that the auxiliary field becomes a two by two matrix $F_{i}{ }^{j}$ which can appear in the following invariant actions;

$$
\widetilde{\mathcal{S}}_{F M}=\int d \tau\left[e^{-1} i 2 \eta_{i}^{k} \mathcal{D}_{\tau} \eta_{i}^{k}+e^{-1} F_{i}^{k} F_{i}^{k}+i 2 \chi^{K}\left(\mathrm{R}_{K}\right)_{\hat{i}}^{j} \eta_{\hat{i}}^{k} F_{j}^{k}\right],
$$

and

$$
\mathcal{S}_{\text {Cosm }}=m_{0} \int d \tau\left[e^{-1} F_{i}^{i}-i 2 \chi^{K}\left(\mathrm{~L}_{K}\right)_{i}{ }^{\hat{j}} \eta_{\hat{j}}^{i}\right]
$$

Finally, there is a term describing an interaction between a scalar multiplet and a single spinor multiplet

$$
\mathcal{S}_{\text {Int }}=\int d \tau\left[e^{-1} F_{i} \mathcal{D}_{\tau} \phi_{i}+i 2 e^{-1} \eta_{\hat{i}} \mathcal{D}_{\tau} \psi_{\hat{i}}+i 2 \chi^{K}\left(\mathrm{R}_{K}\right)_{\hat{i}}^{j} \eta_{\hat{i}} \mathcal{D}_{\tau} \phi_{j}\right] .
$$


We first consider the case of the massless spinning particle with arbitrary Nextended supersymmetry in a second order formalism. The action for such a theory is precisely $\mathcal{S}_{S M}$. The scalar field $\phi_{i}(\tau)$ represents the coordinate of the particle in a d-dimensional spacetime. The quantity $\psi_{\hat{i}}(\tau)$ is the NSR fermion. The first order formalism is obtained by taking as an action $\mathcal{S}_{\text {Int }}-\frac{1}{2} \mathcal{S}_{F M}$. One of the interesting points of the first order formalism is that the auxiliary field of the spinor multiplet, $F_{i}(\tau)$, plays the role of the momentum canonically conjugate to the coordinate $\phi_{i}(\tau)$. Thus, the entire spinor multiplet $\left(\eta_{\hat{i}}, F_{i}\right)$ is the canonical conjugate to the scalar multiplet $\left(\phi_{i}, \psi_{\hat{i}}\right)$.

On the other hand, the massive versions of the theory are considerably different. To describe this version requires that we actually add to either the first or second order massless actions an additional spinor multiplet action! This mass term is describe by $\mathrm{d}^{-1}\left[\mathcal{S}_{\text {Cosm }}-\frac{1}{2} \widetilde{\mathcal{S}}_{F M}\right]$. The equation of motion for $F_{i}{ }^{i}$ sets it equal to the mass parameter $m_{0}$. Without the extra spinor multiplet $\left(\eta_{\hat{k}}{ }^{j}, F_{i}^{j}\right)$, it is not possible to introduce the mass. Finally note that the twisted version of all of the above actions can also be easily constructed.

\section{Conclusions and Summary}

We have shown that it is possible to construct spinning particles with arbitrary numbers of supersymmetries on the world-line. This result has a number of interesting implications. For example, the $\mathrm{N}=1$ and $\mathrm{N}=2 \mathrm{SKdV}$ systems have been shown to arise precisely by starting from the massive spinning particle action and performing a field redefinition on the equation of motion of the scalar multiplet to derive a supersymmetric Lax equation. The implication of our work is that it is possible to infinitely extend the amount of supersymmetry permitted as generalizations of the $\mathrm{KdV}$ system!

Another course that is indicated as a direction of further investigation is the study of utilizing 1D locally supersymmetric systems in descriptions of proposed hyperbolic Kac-Moody algebras as first envisioned by Julia. Here our results for $\mathrm{N}=16$ may be taken as a point of departure. The topic of locally supersymmetric non-linear 1D sigma-models is clearly one that can now be studied in a much more systematic manner. Finally, the interesting question arises as to whether it is possible to take the $\mathrm{N} \rightarrow \infty$ limit of these $1 \mathrm{D}$ models? If this is possible, do we find a new super Kac-Moody algebra? 


\section{APPENDIX A: Explicit L and R Matrix Representations}

In the text of this paper, we have seen that the problem of constructing the $1 \mathrm{D}$, $\mathrm{N}$-extended spinning particle action reduces to finding real matrix solutions to the equations of (3.3) and (3.8).

A more complete discussion of the existence of solutions for these conditions in general will be included in a forthcoming work [7. Here we provide some examples of solutions to these conditions for values up to $\mathrm{N}=16$.

For $\mathrm{N}=2$, there exist a set of two by two matrices:

$$
\mathrm{L}_{1}=i \sigma^{2}=\mathrm{R}_{1} \quad ; \quad \mathrm{L}_{2}=-\mathrm{I}=-\mathrm{R}_{2} .
$$

For $\mathrm{N}=4$, there are two distinct minimal set of matrices that realize the algebra and are well known in the physics literature

$$
\begin{aligned}
& \mathrm{L}_{1}=i \sigma^{1} \otimes \sigma^{2}=\mathrm{R}_{1} \quad ; \quad \widehat{\mathrm{L}}_{1}=i \sigma^{2} \otimes \sigma^{3}=\widehat{\mathrm{R}}_{1} ; \\
& \mathrm{L}_{2}=i \sigma^{2} \otimes \mathrm{I}=\mathrm{R}_{2} ; \quad \widehat{\mathrm{L}}_{2}=-i \mathrm{I} \otimes \sigma^{2}=\widehat{\mathrm{R}}_{2} ; \\
& \mathrm{L}_{3}=-i \sigma^{3} \otimes \sigma^{2}=\mathrm{R}_{3} ; \quad \widehat{\mathrm{L}}_{3}=i \sigma^{2} \otimes \sigma^{1}=\widehat{\mathrm{R}}_{3} ; \\
& \mathrm{L}_{4}=\mathrm{I} \otimes \mathrm{I}=-\mathrm{R}_{4} ; \quad \widehat{\mathrm{L}}_{4}=\mathrm{I} \otimes \mathrm{I}=-\widehat{\mathrm{R}}_{4} .
\end{aligned}
$$

These provide an example of a set of inequivalent representations. Any three within a given set can be used to cover the case of $\mathrm{N}=3$.

For $\mathrm{N}=8$, a convenient set for our required matrices is given by,

$$
\begin{aligned}
& \mathrm{L}_{1}=i \mathrm{I} \otimes \sigma^{3} \otimes \sigma^{2}=\mathrm{R}_{1} ; \quad \mathrm{L}_{5}=i \sigma^{2} \otimes \mathrm{I} \otimes \sigma^{1}=\mathrm{R}_{5} ; \\
& \mathrm{L}_{2}=i \sigma^{3} \otimes \sigma^{2} \otimes \mathrm{I}=\mathrm{R}_{2} ; \quad \mathrm{L}_{6}=i \sigma^{2} \otimes \mathrm{I} \otimes \sigma^{3}=\mathrm{R}_{6} ; \\
& \mathrm{L}_{3}=i \mathrm{I} \otimes \sigma^{1} \otimes \sigma^{2}=\mathrm{R}_{3} ; \quad \mathrm{L}_{7}=i \sigma^{2} \otimes \sigma^{2} \otimes \sigma^{2}=\mathrm{R}_{7} ; \\
& \mathrm{L}_{4}=i \sigma^{1} \otimes \sigma^{2} \otimes \mathrm{I}=\mathrm{R}_{4} ; \quad \mathrm{L}_{8}=\mathrm{I} \otimes \mathrm{I} \otimes \mathrm{I}=-\mathrm{R}_{8} .
\end{aligned}
$$

$\mathrm{N}=5,6$, and 7 can be formed by taking any set of 5,6 or 7 of the $\mathrm{N}=8$ matrices, respectively.

For $\mathrm{N}=9$, we find the matrices to be $16 \times 16$ :

$$
\begin{aligned}
& L_{1}=i \mathrm{I} \otimes \sigma^{3} \otimes \sigma^{2} \otimes \sigma^{1}=R_{1} \\
& L_{2}=i \sigma^{3} \otimes \sigma^{2} \otimes \mathrm{I} \otimes \mathrm{I}=R_{2} \\
& L_{3}=i \mathrm{I} \otimes \sigma^{1} \otimes \sigma^{2} \otimes \sigma^{1}=R_{3} \\
& L_{4}=i \sigma^{1} \otimes \sigma^{2} \otimes \mathrm{I} \otimes \mathrm{I}=R_{4} \\
& L_{5}=i \sigma^{2} \otimes \mathrm{I} \otimes \sigma^{1} \otimes \sigma^{1}=R_{5} \\
& L_{6}=i \sigma^{2} \otimes \mathrm{I} \otimes \sigma^{3} \otimes \sigma^{1}=R_{6} \\
& L_{7}=i \sigma^{2} \otimes \sigma^{2} \otimes \sigma^{2} \otimes \sigma^{1}=R_{7} \\
& L_{8}=i \sigma^{2} \otimes \mathrm{I} \otimes \mathrm{I} \otimes \sigma^{3}=R_{8} \\
& L_{9}=\mathrm{I} \otimes \mathrm{I} \otimes \mathrm{I} \otimes \mathrm{I}=-R_{9}
\end{aligned}
$$


For $\mathrm{N}=10$, we have been able to find a $32 \times 32$ representation:

$$
\begin{aligned}
& \mathrm{L}_{1}=i \sigma^{2} \otimes \mathrm{I} \otimes \mathrm{I} \otimes \mathrm{I} \otimes \mathrm{I}=\mathrm{R}_{1} ; \\
& \mathrm{L}_{2}=i \sigma^{1} \otimes \sigma^{2} \otimes \mathrm{I} \otimes \mathrm{I} \otimes \mathrm{I}=\mathrm{R}_{2} ; \\
& \mathrm{L}_{3}=i \sigma^{1} \otimes \sigma^{1} \otimes \sigma^{2} \otimes \mathrm{I} \otimes \mathrm{I}=\mathrm{R}_{3} ; \\
& \mathrm{L}_{4}=i \sigma^{1} \otimes \sigma^{3} \otimes \sigma^{2} \otimes \mathrm{I} \otimes \mathrm{I}=\mathrm{R}_{4} ; \\
& \mathrm{L}_{5}=i \sigma^{3} \otimes \mathrm{I} \otimes \sigma^{2} \otimes \sigma^{1} \otimes \mathrm{I}=\mathrm{R}_{5} ; \\
& \mathrm{L}_{6}=i \sigma^{3} \otimes \mathrm{I} \otimes \sigma^{2} \otimes \sigma^{3} \otimes \mathrm{I}=\mathrm{R}_{6} ; \\
& \mathrm{L}_{7}=i \sigma^{3} \otimes \mathrm{I} \otimes \mathrm{I} \otimes \sigma^{2} \otimes \sigma^{1}=\mathrm{R}_{7} ; \\
& \mathrm{L}_{1}=i \sigma^{3} \otimes \mathrm{I} \otimes \sigma^{2} \otimes \sigma^{2} \otimes \sigma^{2}=\mathrm{R}_{8} ; \\
& \mathrm{L}_{9}=i \sigma^{3} \otimes \mathrm{I} \otimes \mathrm{I} \otimes \sigma^{2} \otimes \sigma^{3}=\mathrm{R}_{9} ; \\
& \mathrm{L}_{10}=\mathrm{I} \otimes \mathrm{I} \otimes \mathrm{I} \otimes \mathrm{I} \otimes \mathrm{I}=-\mathrm{R}_{10} ;
\end{aligned}
$$

$\mathrm{N}=12$ is a $64 \times 64$ representation:

$$
\begin{aligned}
& L_{1}=i \sigma^{2} \otimes \mathrm{I} \otimes \mathrm{I} \otimes \mathrm{I} \otimes \mathrm{I} \otimes \mathrm{I}=\mathrm{R}_{1} \\
& \mathrm{~L}_{2}=i \sigma^{1} \otimes \sigma^{2} \otimes \mathrm{I} \otimes \mathrm{I} \otimes \mathrm{I} \otimes \mathrm{I}=\mathrm{R}_{2} \\
& \mathrm{~L}_{3}=i \sigma^{1} \otimes \sigma^{1} \otimes \sigma^{2} \otimes \mathrm{I} \otimes \mathrm{I} \otimes \mathrm{I}=\mathrm{R}_{3} ; \\
& \mathrm{L}_{4}=i \sigma^{1} \otimes \sigma^{3} \otimes \sigma^{2} \otimes \mathrm{I} \otimes \mathrm{I} \otimes \mathrm{I}=\mathrm{R}_{4} ; \\
& \mathrm{L}_{5}=i \sigma^{3} \otimes \mathrm{I} \otimes \sigma^{2} \otimes \sigma^{1} \otimes \mathrm{I} \otimes \mathrm{I}=\mathrm{R}_{5} ; \\
& \mathrm{L}_{6}=i \sigma^{3} \otimes \mathrm{I} \otimes \sigma^{2} \otimes \sigma^{3} \otimes \mathrm{I} \otimes \mathrm{I}=\mathrm{R}_{6} ; \\
& \mathrm{L}_{7}=i \sigma^{3} \otimes \mathrm{I} \otimes \mathrm{I} \otimes \sigma^{2} \otimes \sigma^{1} \otimes \mathrm{I}=\mathrm{R}_{7} ; \\
& \mathrm{L}_{8}=i \sigma^{3} \otimes \mathrm{I} \otimes \mathrm{I} \otimes \sigma^{2} \otimes \sigma^{3} \otimes \mathrm{I}=\mathrm{R}_{8} ; \\
& \mathrm{L}_{9}=i \sigma^{3} \otimes \mathrm{I} \otimes \mathrm{I} \otimes \sigma^{2} \otimes \sigma^{2} \otimes \sigma^{2}=\mathrm{R}_{9} ; \\
& \mathrm{L}_{10}=i \sigma^{3} \otimes \mathrm{I} \otimes \sigma^{2} \otimes \sigma^{2} \otimes \sigma^{2} \otimes \sigma^{1}=\mathrm{R}_{10} ; \\
& \mathrm{L}_{11}=i \sigma^{3} \otimes \mathrm{I} \otimes \sigma^{2} \otimes \sigma^{2} \otimes \sigma^{2} \otimes \sigma^{3}=\mathrm{R}_{11} ; \\
& \mathrm{L}_{12}=\mathrm{I} \otimes \mathrm{I} \otimes \mathrm{I} \otimes \mathrm{I} \otimes \mathrm{I} \otimes \mathrm{I}=-\mathrm{R}_{12} ;
\end{aligned}
$$

The case of $\mathrm{N}=11$ is contained as a subset.

At this point we should mention that real Dirac gamma matrices have been discussed in a number of references. However, we found a previous work by De Crumbrugghe and Rittenberg [8] very useful in our study and construction of real generalized Pauli matrix representations. The interested reader is referred to this prior work for additional information.

The final explicit result that we present is for $\mathrm{N}=16$, where we have a $128 \times 128$ 
representation of the $\mathrm{N}=16$ supersymmetry algebra:

\begin{tabular}{|c|c|c|c|c|c|c|c|c|c|c|c|c|c|c|c|}
\hline$=$ & $=i \sigma^{2}$ & $\otimes$ & I & $\otimes$ & $\mathrm{I}$ & $\otimes$ & I & $\otimes$ & I & $\otimes$ & I & $\otimes$ & I & $=$ & $\mathrm{R}_{1}$ \\
\hline$=$ & $=i \sigma^{1}$ & $\otimes$ & $\sigma^{1}$ & $\otimes$ & $\sigma^{2}$ & $\otimes$ & I & $\otimes$ & I & $\otimes$ & I & $\otimes$ & I & $=$ & $\mathrm{R}_{2}$ \\
\hline$=$ & $i \sigma^{1}$ & $\otimes$ & I & $\otimes$ & $\sigma^{1}$ & $\otimes$ & $\sigma^{2}$ & $\otimes$ & I & $\otimes$ & I & ه & I & $=$ & $\mathrm{R}_{3}$ \\
\hline$=$ & $=i \sigma^{1}$ & $\otimes$ & $\mathrm{I}$ & $\otimes$ & $\sigma^{3}$ & $\otimes$ & $\sigma^{2}$ & $\otimes$ & I & $\otimes$ & I & $\theta$ & I & $=$ & $\mathrm{R}_{4}$ \\
\hline$=$ & $=i \sigma^{1}$ & $\otimes$ & $\sigma^{2}$ & $\otimes$ & I & $\otimes$ & $\sigma^{1}$ & $\otimes$ & I & & I & A & I & $=$ & $\mathrm{R}_{5}$ \\
\hline$=$ & $i \sigma^{1}$ & $\otimes$ & $\sigma^{2}$ & $\otimes$ & I & $\otimes$ & $\sigma^{3}$ & 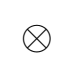 & I & $\otimes$ & I & 8 & I & $=$ & $\mathrm{R}_{6}$ \\
\hline$=$ & $i \sigma^{1}$ & $\otimes$ & $\sigma^{2}$ & $\otimes$ & $\sigma^{2}$ & $\otimes$ & $\sigma^{2}$ & $\otimes$ & I & $\otimes$ & I & A & I & $=$ & $\mathrm{R}_{7}$ \\
\hline$=$ & $=i \sigma^{1}$ & $\otimes$ & $\sigma^{3}$ & $\otimes$ & $\sigma^{2}$ & $\otimes$ & $\mathrm{I}$ & $e$ & 1 & & I & 8 & $\mathrm{I}$ & $=$ & $\mathrm{R}_{8}$ \\
\hline$=$ & $i \sigma^{3}$ & $\otimes$ & $\mathrm{I}$ & $\otimes$ & $\mathrm{I}$ & $\otimes$ & I & $\otimes$ & $\sigma^{2}$ & 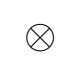 & $\sigma^{2}$ & $\otimes$ & $\sigma^{2}$ & $=$ & $\mathrm{R}_{9}$ \\
\hline$=$ & $i \sigma^{3}$ & $\otimes$ & I & $\otimes$ & I & $\otimes$ & I & $\otimes$ & $\sigma^{2}$ & 0 & I & $\otimes$ & $\sigma^{3}$ & $=$ & $\mathrm{R}_{10}$ \\
\hline$=$ & $i \sigma^{3}$ & $\otimes$ & I & $\otimes$ & I & $\otimes$ & I & $\otimes$ & $\sigma^{2}$ & $\otimes$ & I & $\otimes$ & $\sigma^{1}$ & $=$ & $\mathrm{R}_{11}$ \\
\hline$=$ & $i \sigma^{3}$ & $\otimes$ & I & $\otimes$ & I & $\otimes$ & I & $\otimes$ & $\sigma^{1}$ & P & $\sigma^{2}$ & $\otimes$ & $\mathrm{I}$ & $=$ & $\mathrm{R}_{12}$ \\
\hline$=$ & $i \sigma^{3}$ & $\otimes$ & I & $\otimes$ & I & $\otimes$ & I & $\otimes$ & I & $\otimes$ & $\sigma^{1}$ & $\otimes$ & $\sigma^{2}$ & $=$ & $\mathrm{R}_{13}$ \\
\hline$=$ & $i \sigma^{3}$ & $\otimes$ & I & $\otimes$ & I & $\otimes$ & I & $\otimes$ & $\sigma^{3}$ & 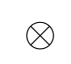 & $\sigma^{2}$ & $\otimes$ & I & $=$ & $\mathrm{R}_{14}$ \\
\hline$=$ & $i \sigma^{3}$ & $\otimes$ & I & $\otimes$ & I & $\otimes$ & I & $\otimes$ & I & & $\sigma^{3}$ & $\otimes$ & $\sigma^{2}$ & $=$ & $\mathrm{R}_{15}$ \\
\hline$=$ & I & $\otimes$ & I & $\otimes$ & I & Q & I & & $\mathrm{I}$ & & I & & I & $=$ & $\mathrm{B}$ \\
\hline
\end{tabular}

Similarly, $\mathrm{N}=13,14$ and 15 can be formed using the matrices for the $\mathrm{N}=16$ case.

\section{APPENDIX B: A Universal Theory of On-Shell D $\leq 3$ Scalar Multiplets}

One of the most interesting implications of our 1D results is that they provide a general way to describe all supersymmetric scalar multiplets for $\mathrm{D} \leq 3$ ! This includes the cases of scalar multiplets in $\mathrm{D}=3, \mathrm{D}=2$ (both heterotic and non-heterotic) and $\mathrm{D}=1$. We now as well have, from the application of our work on fermionic multiplets, a recipe for describing heterotic fermion multiplets for arbitrary N. Below for the scalar multiplets, we explicitly consider the case in 3D (all other cases can be obtained via truncations).

Let $\mathcal{A}_{i}(x)$ and $\psi_{\alpha \hat{j}}(x)$ correspond to a multiplet of real spin-0 and 3D, Majorana spinors, respectively. We propose a set of on-shell supersymmetry variations given by,

$$
\delta_{Q} \mathcal{A}_{i}=\epsilon^{\alpha I}\left(\mathrm{~L}_{I}\right)_{i}{ }^{\hat{j}} \psi_{\alpha \hat{j}} \quad, \quad \delta_{Q} \psi_{\alpha \hat{i}}=i \epsilon^{\beta I}\left(\mathrm{R}_{I}\right)_{\hat{i}}{ }^{j}\left(\gamma^{a}\right)_{\alpha \beta} \partial_{a} \mathcal{A}_{j}
$$

in terms of a constant 3D Majorana spinor parameter $\epsilon^{\alpha K}$. (Note that our convention for complex conjugation of spinors is: $\left(\epsilon^{\alpha}\right)^{*}=+\epsilon^{\alpha}$ and $\left(\epsilon_{\alpha}\right)^{*}=-\epsilon_{\alpha}$.) A simple calculation reveals,

$$
\left[\delta_{Q}\left(\epsilon_{1}\right), \delta_{Q}\left(\epsilon_{2}\right)\right] \mathcal{A}_{i}=\widehat{\xi}_{12}^{a} \partial_{a} \mathcal{A}_{i}
$$


where $\widehat{\xi}_{12}^{a} \equiv i 2 \epsilon_{1}^{\alpha I} \epsilon_{2}^{\beta I}\left(\gamma^{a}\right)_{\alpha \beta}$. Similarly, we have for the spinor field

$$
\left[\delta_{Q}\left(\epsilon_{1}\right), \delta_{Q}\left(\epsilon_{2}\right)\right] \psi_{\alpha \hat{i}}=\widehat{\xi}_{12}^{a} \partial_{a} \psi_{\alpha \hat{i}}+i \frac{1}{2} \epsilon_{\alpha[1}^{I} \epsilon_{2]}^{\beta J}\left(\mathrm{R}_{J} \mathrm{~L}_{I}\right)_{\hat{i}}^{\hat{j}}\left(\gamma^{a}\right)_{\beta \gamma} \partial_{a} \psi_{\hat{j}}^{\gamma}
$$

The latter term on the RHS of (B.3) is proportional to the equation of motion for the spinor field and in an on-shell description we set it equal to zero as is customary. The invariant on-shell action takes the expected form,

$$
\mathcal{S}_{3 D, S M}=\int d^{3} x \frac{1}{2}\left[\eta^{a b}\left(\partial_{a} \mathcal{A}_{i}\right)\left(\partial_{b} \mathcal{A}_{i}\right)+i\left(\gamma^{a}\right)^{\beta \gamma} \psi_{\beta \hat{j}} \partial_{a} \psi_{\gamma \hat{j}}\right]
$$

We should mention that for 3D theories, supersymmetric scalar multiplets are dual to supersymmetric vector multiplets. So our work as well provides a basis for construction of 3D, arbitrary N-extended supersymmetric vector multiplets described by fields $\left(\mathcal{B}_{i}{ }^{j}, \lambda_{\alpha \hat{k}}{ }^{i}, A_{a}\right)$. These have variations given by,

$$
\begin{aligned}
\delta_{Q} \mathcal{B}_{i}{ }^{j} & =\epsilon^{\alpha I}\left(\mathrm{~L}_{I}\right)_{k}{ }^{\hat{k}}\left[\delta_{i}{ }^{k} \lambda_{\alpha \hat{k}}{ }^{j}-\mathrm{d}^{-1} \delta_{i}{ }^{j} \lambda_{\alpha \hat{k}}{ }^{k}\right] \\
\delta_{Q} \lambda_{\alpha \hat{k}}{ }^{k} & =i \epsilon^{\beta I}\left(\mathrm{R}_{I}\right)_{\hat{k}}{ }^{j}\left(\gamma^{a}\right)_{\alpha \beta}\left[\partial_{a} \mathcal{B}_{j}{ }^{k}+\frac{1}{2} \mathrm{~d}^{-1} \delta_{j}{ }^{k} \epsilon_{a}{ }^{b c} F_{b c}\right] \\
\delta_{Q} A_{a} & =i \epsilon^{\alpha I}\left(\mathrm{~L}_{I}\right)_{k}{ }^{\hat{k}}\left(\gamma_{a}\right)_{\alpha \beta} \lambda_{\hat{k}}^{\beta}
\end{aligned}
$$

The spectrum as well as the supersymmetry variations are completely determined by tensoring the $i$ representation with (B.1) and then performing a duality transformation only on the resultant singlet spin-0 field. It is also possible to form a twisted version of both the N-extended scalar and vector multiplets.

Thus, we see that the equations in (3.3) together with (3.8) provide a universal formalism for describing on-shell scalar multiplet theories in $\mathrm{D} \leq 3$ for all values of $\mathrm{N}$ (the degree of extended supersymmetry). We believe that this work is also of great importance for future studies. The search for the off-shell versions of these theories is clearly a topic that warrants study. Such work may ultimately shed light on the off-shell problem for even higher dimensional theories. Once again, we cannot help but wonder about the nature of the $\mathrm{N} \rightarrow \infty$ limit of the $3 \mathrm{D}$ theories. According to at least one previous work on supersymmetric quantum mechanical systems [8], Nextended supersymmetry imposes a constraint such that for $\mathrm{N}>4$ the Hamiltonian is integrable! 


\section{References}

[1] A. Barducci, R. Casalbuoni, and L. Lusanna, Nuovo Cim. 35 (1976) 377; L. Brink, S. Deser, B. Zumino, P. Di Vecchia and P. Howe, Phys. Lett. 64B (1976) 435; P. Collins and R. Tucker, Nucl Phys. B121 (1977) 307.

[2] See for example, W. Siegel, Introduction to String Field Theory, World Scientific, 1988.

[3] l Alvarez-Gaumé, Commun. Math. Phys. 90 (1983) 161.

[4] B. Julia, Superspace and Supergravity, eds. S. Hawking and M. Roček, Cambridge Univ. Press, 1981; Lect. in App. Math. 21 (1985) 355; H. Nicolai, Phys. Lett. 275B (1992) 333; B. Julia and H. Nicolai, "Null-Killing Vector Dimensional Reduction and Galilean Geometodynamics", preprint LPTEN 94/221, DESY 94-156, 1994.

[5] J. Figueroa-O'Farrill and E. Ramos, Commun.Math. Phys. 145 (1992) 43; E. Ramos and J. Roca, "W-symmetry and the Rigid Particle", preprint QMWPH-94-23, hep-th/9408019, 1994.

[6] S. Gates and L. Rana, Phys. Lett. 345 (1995) 233.

[7] S. Gates and L. Rana, "On Extended Supersymmetric Quantum Mechanics", Univ. of Maryland preprint UMDPP 93-194, in progress.

[8] M. De Crombrugghe and V. Rittenberg, Ann. of Phys. 151 (1983) 99. 